\title{
Priorización de enfermedades virales zoonóticas en la interfaz de cerdos silvestres, cerdos domésticos y seres humanos
}

\author{
Diana Benavides-Arias ${ }^{1}$, Diego Soler-Tovar² \\ 1 Semillero de Investigación Una Salud, Programa de Medicina Veterinaria, Facultad de Ciencias Agropecuarias, \\ Universidad de La Salle, Bogotá, D.C., Colombia \\ 2 Grupo de Epidemiología y Salud Pública, Facultad de Ciencias Agropecuarias, Universidad de La Salle, \\ Bogotá, D.C., Colombia
}

Introducción. Para entender la ecología de las enfermedades es necesario comprender los agentes patógenos en la interfaz de vida silvestre y ganado. Los cerdos silvestres (Sus scrofa) constituyen un problema sanitario cuando se trata de prevenir y controlar las enfermedades zoonóticas, pues en ocasiones sus poblaciones son portadores de agentes infecciosos transmisibles a los cerdos domésticos y a otras especies animales, incluidos los seres humanos.

Objetivo. Priorizar las enfermedades zoonóticas en la interfaz de cerdos silvestres, animales domésticos y seres humanos.

Materiales y métodos. Se utilizó el método de priorización semicuantitativa basado en 27 criterios sustentados en publicaciones recientes, los cuales se clasificaron en las siguientes cinco categorías con base en la etiología viral: epidemiología (ocho), prevención y control (tres), economía y comercio (cuatro), salud pública (nueve) y sociedad (tres). A cada criterio se le adjudicó un coeficiente entre 0 y 7 de acuerdo con su impacto medido con base en la información científica (suma total de 189). La información sobre los criterios para las nueve enfermedades virales analizadas se recolectó mediante la revisión de 81 fuentes publicadas entre 1977 y 2015.

Resultados. Las tres enfermedades con mayor puntaje y potencial zoonótico fueron la influenza porcina (133), la hepatitis E (123) y la infección por hantavirus (103), y la mayor puntuación se observó en los criterios de epidemiología y salud pública.

Conclusión. Los métodos semicuantitativos de priorización son una fuente de información para la toma de decisiones, pero su utilización es poco frecuente en los países en desarrollo por la falta de datos de vigilancia en salud pública. El control de las enfermedades que afectan tanto a los seres humanos como a los animales silvestres, requiere el desarrollo de estrategias que reduzcan la transmisión de patógenos de estos a los animales domésticos y a los seres humanos.

Palabras clave: ecología, Sus scrofa, virus, zoonosis.

doi: http://dx.doi.org/10.7705/biomedica.v36i0.2950

Prioritization of zoonotic viral diseases in feral pigs, domestic pigs and humans interface

Introduction: Understanding the ecology of diseases requires the comprehension of pathogens in wild life-livestock interface. Feral pigs (Sus scrofa) are a health problem when countries work to prevent and control zoonotic diseases, as their populations raise environmental and health concerns due to infectious agents transmissible to domestic pigs and other animal species, including humans.

Objective: To prioritize zoonotic diseases in the feral pigs, domestic animals and humans interface.

Materials and methods: The semi-quantitative prioritization method based on evidence included 27 criteria founded in recent publications. According to viral etiology we classified them in five categories: epidemiology (eight), prevention/control (three), economy/trade (four), public health (nine) and society (three). Each criterion had a coefficient of 0 to 7 according to their impact based on evidence (maximum sum of 189). Evidence on the criteria for the nine viral diseases analyzed came from the review of 81 sources published between 1977 and 2015.

Results: The top three diseases with the highest score and zoonotic potential were swine influenza (133), hepatitis E (123), and hantavirus infection (103), whose highest scores were observed on epidemiology and public health criteria.

\footnotetext{
Contribución de los autores:

Todos los autores participaron en las diferentes fases del desarrollo del estudio y en la redacción del artículo.
} 
Conclusion: The semi-quantitative methods of prioritization impartially contribute to decision-making based on evidence; however, they are seldom used in developing countries due to the lack of data from public health surveillance. Control of shared diseases requires the development of strategies to reduce transmission of pathogens between wildlife and domestic animals and humans.

Key words: Ecology, Sus scrofa, viruses, zoonoses. doi: http://dx.doi.org/10.7705/biomedica.v36i0.2950

El control de las enfermedades infecciosas se dificulta por los escasos estudios sobre los procesos ecológicos que favorecen la transmisión entre los reservorios de fauna silvestre y las poblaciones de ganado, y la falta de comprensión por parte de los epidemiólogos y los responsables de las políticas de las complejas interacciones entre especies en los potenciales huéspedes de agentes patógenos y de la identificación de los factores de riesgo de enfermedades asociados a ellos. Para entender la ecología de las enfermedades, es necesario comprender los agentes patógenos involucrados en la interfaz de la vida silvestre y el ganado. La importancia de evaluar cómo las especies animales utilizan el entorno y el impacto de sus interacciones, es un factor determinante para establecer el riesgo local en la transmisión y la persistencia de las enfermedades infecciosas (1).

Debido a los brotes de enfermedades en especies amenazadas y la consecuente presencia de veterinarios, así como a los avances de la biología y la interacción entre huéspedes y parásitos, actualmente se presta mayor atención a las enfermedades de la fauna que constituyen una amenaza para los humanos, lo cual favorece los estudios encaminados a conocer mejor la interfaz del cerdo doméstico, el cerdo salvaje y los seres humanos (2).

Por otra parte, la mayoría de los agentes patógenos que afectan el ganado son capaces de infectar a otras especies de huéspedes, incluidas las de los animales de vida silvestre, por lo cual en las zonas donde la fauna silvestre y el ganado coexisten, dichos agentes patógenos pueden surgir y establecerse (3).

De igual manera, las enfermedades emergentes y reemergentes que afectan a los animales silvestres pueden transmitirse a las poblaciones humanas por contacto directo o por la acción de los vectores

Correspondencia:

Diana Benavides-Arias, Semillero de Investigación Una Salud, Universidad de La Salle, Carrera 7 № 172-85, Bogotá, D. C.,

Colombia

Teléfono: (311) 5478232

dbenavides29@unisalle.edu.co

Recibido: 02/07/15; aceptado: 03/12/15
(4). Según Gortázar, et al. (3), desde la perspectiva de la salud veterinaria, las enfermedades de notificación obligatoria que implican múltiples huéspedes, y las que han sido erradicadas 0 están casi bajo control, tienen el pronóstico más desfavorable; el contacto de animales silvestres con el ganado produce graves consecuencias para la salud y la economía.

Los procesos de priorización son una herramienta desarrollada específicamente para satisfacer las necesidades de las personas que trabajan en zonas donde los datos cuantitativos sobre las zoonosis son escasos y los vínculos entre los encargados de la salud humana y los responsables de la salud no operan de forma óptima. Los métodos semicuantitativos, cuyo uso depende de las preferencias individuales, ofrecen opciones para la clasificación mediante una escala numérica, en tanto que los métodos cuantitativos se basan en escalas numéricas diseñadas para reflejar los valores objetivos (por ejemplo, prevalencia o incidencia). Estos métodos, utilizados por diversos grupos de interés en países desarrollados, proporcionan una lista dinámica de zoonosis prioritarias que pueden ser objeto de investigación y de la asignación de fondos en busca del fortalecimiento de la colaboración entre las diversas partes interesadas en la prevención del riesgo (5).

La mayoría de las enfermedades infecciosas emergentes o reemergentes en los países desarrollados y en desarrollo, se origina en los animales, con más de 250 zoonosis documentadas en la literatura científica (6). Además de la aparición de agentes patógenos zoonóticos, se estima que $20 \%$ de la morbimortalidad de todas las enfermedades humanas en los países menos desarrollados es atribuible a las zoonosis endémicas (7). En los países en desarrollo, 13 zoonosis se consideran como las de mayor impacto en los ganaderos pobres, con el agravante de que las enfermedades zoonóticas son un problema de salud pública, y se estima que son responsables de 2,7 millones de muertes y 2,4 millones de casos de enfermedad en seres humanos cada año; la mayoría de estas enfermedades también tienen efectos negativos sobre la producción ganadera (5). 
El objetivo del presente estudio fue priorizar las enfermedades zoonóticas virales en la interfaz de cerdos silvestres (Sus scrofa), cerdos domésticos y seres humanos en Colombia, mediante una revisión sistemática de los sistemas de indexación y resumen (SIRES) en Colombia y de los datos del Sistema Mundial de Información Zoosanitaria (World Animal Health Information Database, WAHID, y World Animal Health Information System, WAHIS), utilizando el método semicuantitativo de priorización basado en la evidencia.

\section{Materiales y métodos}

Mediante la consulta en el Sistema Mundial de Información Zoosanitaria (WAHID y WAHIS) (http://www.oie.int/es/sanidad-animal-en-elmundo/el-sistema-mundial-de-informacionsanitaria/datos-despues-2004-wahid/; http://www. oie.int/es/sanidad-animal-en-el-mundo/wahiswild-interface/), y en los sistemas de indexación y resúmenes (SIRES) Science Direct, Scopus, Google Scholar y PubMed (los cuales contenían información para la construcción de la matriz de categorías y criterios de priorización), de artículos indexados entre 1977 y 2015, y usando las palabras clave "priorización", "cerdos silvestres" y "enfermedades virales" en inglés y en español, se indagó sobre las enfermedades zoonóticas y se elaboró una lista de nueve en las cuales los cerdos silvestres están involucrados como huéspedes o reservorios. La priorización semicuantitativa basada en la evidencia de las enfermedades virales se hizo según Humblet, et al. (8), Logan, et al. (5), y Cito, et al. (9), y se confrontó la información obtenida sobre las enfermedades zoonóticas de importancia a nivel internacional para detectar las más comunes.

Con base en la consulta documental, las enfermedades virales incluidas en la priorización fueron: la circovirosis porcina, la hepatitis E, la enfermedad de Aujeszky (pseudorrabia), la peste porcina clásica, la parvovirosis, el síndrome reproductivo y respiratorio porcino (Porcine Reproductive and Respiratory Syndrome, PRRS), la peste porcina africana, la infección por hantavirus y la influenza porcina.

La distribución de los criterios múltiples entre las cinco categorías establecidas, fue la siguiente: epidemiología (EP), ocho criterios; prevención y control (PC), tres criterios; economía y comercio $(E C)$, cuatro criterios; salud pública $(\mathrm{PH})$, nueve criterios, y sociedad (SO), tres criterios (7) (cuadro 1).

\section{Coeficientes de los criterios}

Se asignó un coeficiente de 0 a 7, adaptado de Humblet, et al. (8), a cada criterio de acuerdo con su función, el efecto o la tasa. Los coeficientes se correlacionaron con la gravedad, es decir, cuanto mayor era el coeficiente, mayor o más grave era el efecto. Por ejemplo, a una tasa de letalidad de menos de $1 \%$, correspondía un coeficiente de 1 , y a una de más de $90 \%$, uno de 7 . A los agentes no zoonóticos se les asignó un coeficiente de 0 , el cual representaba la ausencia de riesgo, en tanto que 1 y 2 correspondían a un riesgo mínimo, los valores intermedios de 3, 4 y 5 representaban riesgo pero sin que implicara peligro grave, y los coeficientes de 6 y 7 representaban la mayor probabilidad de riesgo (cuadro 1).

\section{Resultados}

Mediante la priorización semicuantitativa basada en la evidencia, se dio la puntuación por categoría de múltiples criterios para las enfermedades virales en el cerdo silvestre. En los cuadros 2, 3 y 4 se muestran los puntajes de las enfermedades virales prioritarias, clasificados como superiores, medios $y$ bajos.

Según la priorización, las tres enfermedades zoonóticas con mayor puntuación (cuadros 2 y 5 ) en general y de acuerdo con la categoría (cuadro 6), fueron la influenza porcina (133), la hepatitis E (123) y la infección por hantavirus (103).

Las tres enfermedades priorizadas (influenza, hepatitis $\mathrm{E}$ e infección por hantavirus) presentaron los coeficientes de criterio más altos, con puntajes de 6 y 7, principalmente en las categorías de epidemiología y salud pública, lo cual se relacionó con una mayor exposición a los factores de riesgo que favorecen la presentación de la enfermedad. La priorización basada en la evidencia permitió asignar el coeficiente más alto de acuerdo con los criterios referidos a las características del huésped, a su sensibilidad a un agente patógeno presente en el ambiente, y a su interacción con las demás especies en el entorno.

En el caso de enfermedades como la peste porcina clásica, la circovirosis y la parvovirosis porcina (cuadro 3), mientras que en la categoría de epidemiología se registraron coeficientes entre 6 y 7 , en la de salud pública los valores fueron de 1. Esto podría estar relacionado con los programas de prevención y control de enfermedades que adelantan las autoridades sanitarias en el país mediante estrategias como las zonas libres con vacunación, los programas de vacunación, y otras. 
Cuadro 1. Descripción de las categorías y los criterios para la priorización semicuantitativa

\begin{tabular}{|c|c|c|}
\hline Categoría & Criterio & Descripción \\
\hline \multirow[t]{24}{*}{ Epidemiología } & 1. Tasa de morbilidad & $\begin{array}{l}\text { Ausencia de morbilidad: } 0 \text {; tasa de morbilidad }<1 \% \text { : coeficiente de } 1 \text {; tasa de } \\
\text { morbilidad }<50 \% \text { : coeficiente de } 4 \text {, y tasa de morbilidad }>90 \% \text { : coeficiente de } 7\end{array}$ \\
\hline & 2. Tasa de mortalidad & $\begin{array}{l}\text { Ausencia de mortalidad: } 0 \text {; tasa de mortalidad }<1 \% \text { : coeficiente de } 1 \text {; tasa de } \\
\text { mortalidad }<50 \% \text { : coeficiente de } 4 \text {, y tasa de mortalidad }>90 \% \text { : coeficiente de } 7\end{array}$ \\
\hline & \multirow[t]{6}{*}{ 3. Especificidad del patógeno } & No se encuentra información reportada: 0 . \\
\hline & & Si solo se presenta en el cerdo: 4 \\
\hline & & Si tiene múltiples huéspedes e involucra animales domésticos: 5. \\
\hline & & Si se presenta únicamente en el cerdo o en otras especies: 7. \\
\hline & & Salto entre especies, mayor valor: 7 \\
\hline & & Si involucra animales silvestres: 7. \\
\hline & \multirow[t]{3}{*}{ 4. Modo de transmisión } & Indirecta: 5 \\
\hline & & Horizontal: 7 \\
\hline & & $\begin{array}{l}\text { Vertical: implica periodo de gestación: 6; directa: no necesita huésped } \\
\text { intermediario, vector o reservorio: } 7\end{array}$ \\
\hline & \multirow[t]{3}{*}{ 5. Persistencia en el ambiente } & Sobreviven en el ambiente durante un periodo prolongado. \\
\hline & & Se inactiva rápidamente en el ambiente: 1. \\
\hline & & Están en el ambiente pero sobreviven días: 7. \\
\hline & \multirow[t]{4}{*}{ 6. Enfermedad clínica en cerdos } & Sin signos: 0 \\
\hline & & Portadores: 1 \\
\hline & & Enfermedad subclínica: 4 \\
\hline & & Signos clínicos: 7 \\
\hline & \multirow[t]{4}{*}{ 7. Enfermedad clínica en animales silvestres } & Sin signos: 0 \\
\hline & & Portadores: 1 \\
\hline & & Enfermedad subclínica; 4 \\
\hline & & Signos clínicos: 7 \\
\hline & \multirow[t]{2}{*}{ 8. Evidencia de circulación en Colombia } & No hay datos: 1 . \\
\hline & & Datos epidemiológicos en Colombia: 7 \\
\hline \multirow{7}{*}{$\begin{array}{l}\text { Prevención } \\
\text { y control }\end{array}$} & \multirow[t]{5}{*}{ 1. Control de reservorio y vector } & Asociado a modo de transmisión; para aquellas que tengan un \\
\hline & & modo de transmisión directo, el valor es 0. \\
\hline & & Se hace control efectivo: 1 . \\
\hline & & No se hace control físico o químico del vector o agente: 7 . \\
\hline & & Ningún control de reservorio o vector: 7 \\
\hline & 2. Vacunación & El agente tiene vacuna: 1 ; el agente no tiene vacuna: 7. \\
\hline & 3. Tratamiento & El agente tiene tratamiento: $1 ;$ el agente no tiene tratamiento: 7. \\
\hline \multirow[t]{9}{*}{$\begin{array}{l}\text { Economía } \\
\text { y comercio }\end{array}$} & 1. Disminución de la productividad & $\begin{array}{l}\text { Los animales domésticos no disminuyen su productividad: } 0 \text {. } \\
\text { Los animales domésticos disminuyen su productividad: } 7 \text {. }\end{array}$ \\
\hline & \multirow{3}{*}{$\begin{array}{l}\text { 2. Limitaciones comerciales (importación- } \\
\text { exportación) }\end{array}$} & Prohibición de exportación e importación: 7 \\
\hline & & Impacto no es tan drástico: 4. \\
\hline & & No hay limitación en el comercio exterior: 1. \\
\hline & \multirow[t]{2}{*}{ 3. Impacto en sectores adyacentes (turismo) } & No hay impacto en la industria del turismo: 1. \\
\hline & & Disminución de turistas nacionales e internacionales: 7 \\
\hline & \multirow[t]{3}{*}{ 4. Impacto zoonótico (costo de la enfermedad) } & No hay reportes: 0 . \\
\hline & & No hay evidencia de enfermedades en humanos: 1. \\
\hline & & Elevados costos de enfermedades zoonóticas: 7 \\
\hline \multirow{17}{*}{$\begin{array}{l}\text { Salud } \\
\text { pública }\end{array}$} & \multirow[t]{2}{*}{ 1. Zoonosis } & No es una enfermedad zoonótica: 1. \\
\hline & & Es una enfermedad zoonótica: 7 \\
\hline & 2. Clasificación de la zoonosis & Ciclozoonosis: 4; zooantroponosis: 7; antropozoonosis: 7; metazoonosis: 7 \\
\hline & 3. Enfermedad conocida en humanos & Enfermedad desconocida en humanos: 0 \\
\hline & & Enfermedad conocida en humanos: 7 \\
\hline & 4. Tasa de morbilidad & $\begin{array}{l}\text { No hay morbilidad: } 0 \text {; tasa de morbilidad }<1 \% \text { : coeficiente de } 1 \text {; tasa de } \\
\text { morbilidad }<50 \% \text { : coeficiente de } 4 \text {, y tasa de morbilidad }>90 \% \text { : coeficiente de } 7\end{array}$ \\
\hline & 5. Tasa de mortalidad & $\begin{array}{l}\text { No hay mortalidad: } 0 \text {; tasa de mortalidad }<1 \% \text { : coeficiente de } 1 \text {; tasa de } \\
\text { mortalidad }<50 \% \text { : coeficiente de } 4 \text {, y tasa de mortalidad }>90 \% \text { : coeficiente de } 7\end{array}$ \\
\hline & 6. Modo de transmisión & Indirecta: 5 \\
\hline & & Horizontal: 7 \\
\hline & & Vertical: implica periodo de gestación: 6 \\
\hline & & Directa: no necesita huésped intermediario, vector o reservorio: 7. \\
\hline & 7. Existencia de plan de control & Existe plan de control: 1. \\
\hline & & No existe plan de control: 7 . \\
\hline & 8. Vacunación & Plan de vacunación: 1 \\
\hline & & No tiene plan de vacunación: 7 . \\
\hline & 9. Tratamiento & Se hace tratamiento médico: 1. \\
\hline & & No se hace tratamiento médico: 7 . \\
\hline Sociedad & 1. Disminución del consumo & Disminución del consumo de carne de cerdo: 7 \\
\hline & & No hay impacto negativo en la industria porcina: 1 . \\
\hline & 2. Percepción del problema por parte del & No tiene conocimiento de las enfermedades que afectan al cerdo: 7. \\
\hline & consumidor & Conoce las enfermedades y microorganismos que afectan al cerdo: 1 . \\
\hline & 3. Impacto en animales silvestres y en la & Impacto negativo en los animales silvestres: 7 \\
\hline & biodiversidad & No hay impacto en animales silvestres y biodiversidad: 0 . \\
\hline
\end{tabular}


Cuadro 2. Puntuación alta de enfermedades virales con potencial zoonótico

\begin{tabular}{|c|c|c|c|c|c|c|c|}
\hline Categoría & Criterio & Influenza & Referencia & Hepatitis E & Referencia & $\begin{array}{l}\text { Infección por } \\
\text { hantavirus }\end{array}$ & Referencia \\
\hline & 1. Tasa de morbilidad & 7 & $(10)$ & 7 & (19) & 0 & \\
\hline \multirow[t]{7}{*}{ Epidemiología } & 2. Tasa de mortalidad & 6 & (10) & 1 & (20) & 0 & \\
\hline & 3. Especificidad del patógeno & 7 & (11) & 7 & $(21)$ & 5 & (30) \\
\hline & 4. Modo de transmisión & 7 & (12) & 7 & (21) & 7 & (31) \\
\hline & 5. Persistencia en el medio ambiente & 7 & (13) & 2 & (20) & 7 & (32) \\
\hline & 6. Enfermedad clínica en cerdos & 7 & (11) & 7 & $(22)$ & 2 & $(31)$ \\
\hline & $\begin{array}{l}\text { 7. Enfermedad clínica en animales } \\
\text { silvestres }\end{array}$ & 7 & (11) & 4 & (23) & 5 & (33) \\
\hline & 8. Evidencia de circulación en Colombia & 7 & (14) & 6 & $(24)$ & 5 & $(4)$ \\
\hline Prevención & 1. Control de reservorio y vector & & & 4 & (20) & 6 & (30) \\
\hline \multirow[t]{2}{*}{ y control } & 2. Vacunación & 1 & (15) & 3 & (25) & 7 & (31) \\
\hline & 3. Tratamiento & 0 & & 4 & (25) & 7 & (31) \\
\hline Economía & 1. Disminución de la productividad & 4 & (15) & 1 & (19) & 3 & (31) \\
\hline \multirow[t]{3}{*}{ y comercio } & $\begin{array}{l}\text { 2. Limitaciones de importación- } \\
\text { exportación }\end{array}$ & 6 & (16) & 1 & (19) & 2 & (33) \\
\hline & $\begin{array}{l}\text { 3. Impacto en sectores adyacentes } \\
\text { (turismo) }\end{array}$ & 7 & (11) & 4 & (19) & 4 & (31) \\
\hline & $\begin{array}{l}\text { 6. Impacto zoonótico (costo de la } \\
\text { enfermedad) }\end{array}$ & 7 & (11) & 5 & $(26)$ & 3 & (33) \\
\hline \multirow[t]{9}{*}{ Salud pública } & 1. Zoonosis & 7 & (11) & 7 & $(26)$ & 7 & (34) \\
\hline & 2. Clasificación de la zoonosis & & & 7 & (26) & & \\
\hline & 3. Enfermedad conocida en humanos & 7 & $(12)$ & 7 & $(27)$ & 6 & (33) \\
\hline & 4. Tasa de morbilidad & 2 & (13) & 5 & (28) & 6 & (33) \\
\hline & 5. Tasa de mortalidad & 6 & $(17)$ & 2 & (21) & 5 & (31) \\
\hline & 6. Modo de transmisión & 7 & (12) & 5 & (28) & 7 & (31) \\
\hline & 7. Hay plan de control & 2 & (16) & 4 & (29) & 5 & (31) \\
\hline & 8. Vacunación & 1 & (17) & 1 & (29) & 1 & (31) \\
\hline & 9. Tratamiento & 2 & (18) & 7 & (29) & 2 & (31) \\
\hline \multirow[t]{3}{*}{ Sociedad } & 1. Disminución del consumo & 7 & (16) & 4 & (29) & 0 & \\
\hline & $\begin{array}{l}\text { 2. Percepción del problema por parte del } \\
\text { consumidor }\end{array}$ & 7 & (17) & 6 & (29) & 0 & \\
\hline & $\begin{array}{l}\text { 3. Impacto en animales silvestres y en la } \\
\text { biodiversidad }\end{array}$ & 5 & (11) & 5 & (19) & 1 & $(31)$ \\
\hline Total & & 133 & & 123 & & 103 & \\
\hline
\end{tabular}

Por último, enfermedades como la peste porcina africana, el síndrome respiratorio y reproductivo porcino, y la enfermedad de Aujeszky (cuadro 4), tuvieron coeficientes altos en categorías como la de epidemiología, pero en la categoría de salud pública se registraron valores de 0 , lo cual evidencia que en Colombia estas tres enfermedades representan un riesgo mínimo para los cerdos domésticos, los cerdos silvestres y los seres humanos, ya que la presencia del agente está controlada o es una enfermedad considerada exótica (como es el caso de la peste porcina africana).

\section{Discusión}

La implementación de métodos de priorización para determinar el impacto de las enfermedades virales en la interfaz de cerdos silvestres, animales domésticos y seres humanos, es de vital importancia, ya que esta relación tiene consecuencias directas e indirectas sobre la transmisión de las enfermedades (9). En diversos estudios se ha demostrado la presencia de anticuerpos contra enfermedades virales zoonóticas en poblaciones de cerdos silvestres, entre ellos, contra el virus de la hepatitis $\mathrm{E}$ y el virus de la influenza porcina que, como se sabe, pueden transmitirse a las personas que están en estrecho contacto con estos animales (85).

El propósito de implementar el método de priorización de enfermedades es optimizar los recursos financieros y humanos para la vigilancia, la prevención, el control y la eliminación de las enfermedades infecciosas, en especial, para la detección temprana de cualquier enfermedad emergente, con base en el análisis y la puntuación de múltiples criterios, sobre todo de aquellos que determinan la importancia de los agentes patógenos (86).

En este análisis se incluyeron 27 criterios y en la lista de de enfermedades se incluyeron algunas cuya circulación en el país no está documentada, por ejemplo, la enfermedad de Aujeszky (80) y la peste porcina africana (62), así como tres 
Cuadro 3. Puntuación alta e intermedia de enfermedades virales con presencia en el país

\begin{tabular}{|c|c|c|c|c|c|c|c|}
\hline Categoría & Criterio & $\begin{array}{l}\text { Peste porcina } \\
\text { clásica }\end{array}$ & Referencia & Circovirosis & Referencia & Parvovirosis & Referencia \\
\hline \multirow[t]{8}{*}{ Epidemiología } & 1. Tasa de morbilidad & 3 & (35) & 7 & $(43)$ & 5 & (54) \\
\hline & 2. Tasa de mortalidad & 7 & (36) & 4 & (43) & 3 & (54) \\
\hline & 3. Especificidad del patógeno & 7 & (37) & 5 & (43) & 4 & (55) \\
\hline & 4. Modo de transmisión & 7 & (38) & 7 & (44) & 7 & (56) \\
\hline & $\begin{array}{l}\text { 5. Persistencia en el medio } \\
\text { ambiente }\end{array}$ & 7 & (39) & 7 & (44) & 6 & (55) \\
\hline & 6. Enfermedad clínica en cerdos & 7 & $(40)$ & 7 & $(45)$ & 4 & (57) \\
\hline & $\begin{array}{l}\text { 7. Enfermedad clínica en animales } \\
\text { silvestres }\end{array}$ & 6 & $(40)$ & 5 & (46) & 5 & (58) \\
\hline & 8. Evidencia de circulación en Colombia & 5 & (37) & 7 & $(47)$ & 6 & (59) \\
\hline Prevención & 1. Control de reservorio y vector & 1 & (41) & 0 & (45) & 1 & (56) \\
\hline \multirow[t]{2}{*}{ y control } & 2. Vacunación & 0 & & 1 & (48) & 1 & (60) \\
\hline & 3. Tratamiento & 7 & $(41)$ & 3 & (48) & 5 & (60) \\
\hline Economía & 1. Disminución de la productividad & 5 & $(40)$ & 7 & (49) & 6 & (57) \\
\hline \multirow[t]{3}{*}{ y comercio } & $\begin{array}{l}\text { 2. Limitaciones de importación- } \\
\text { exportación }\end{array}$ & 6 & (42) & 5 & (50) & 7 & (55) \\
\hline & $\begin{array}{l}\text { 3. Impacto en sectores adyacentes } \\
\text { (turismo) }\end{array}$ & 4 & (35) & 2 & $(50)$ & 5 & (58) \\
\hline & $\begin{array}{l}\text { 6. Impacto zoonótico (costo de la } \\
\text { enfermedad) }\end{array}$ & 0 & & 0 & & 1 & $(57)$ \\
\hline Salud & 1. Zoonosis & 0 & & 1 & (51) & 1 & $(57)$ \\
\hline \multirow{8}{*}{ pública } & 2. Clasificación de la zoonosis & 0 & & 1 & (51) & 1 & (57) \\
\hline & 3. Enfermedad conocida en humanos & 1 & $(42)$ & 1 & (52) & 1 & (57) \\
\hline & 4. Tasa de morbilidad & 1 & (35) & 1 & (52) & 1 & (57) \\
\hline & 5. Tasa de mortalidad & 1 & (35) & 1 & (52) & 1 & (57) \\
\hline & 6. Modo de transmisión & 1 & (38) & 1 & (52) & 1 & (57) \\
\hline & 7. Hay plan de control & 1 & (38) & 1 & (52) & 1 & (57) \\
\hline & 8. Vacunación & 1 & (38) & 1 & (52) & 1 & (57) \\
\hline & 9. Tratamiento & 1 & (38) & 1 & (52) & 1 & (57) \\
\hline \multirow[t]{3}{*}{ Sociedad } & 1. Disminución del consumo & 4 & (38) & 4 & (53) & 4 & (58) \\
\hline & $\begin{array}{l}\text { 2. Percepción del problema por parte del } \\
\text { consumidor }\end{array}$ & 5 & (38) & 4 & (53) & 3 & (55) \\
\hline & $\begin{array}{l}\text { 3. Impacto en animales silvestres y en la } \\
\text { biodiversidad }\end{array}$ & 4 & (38) & 7 & $(46)$ & 4 & $(57)$ \\
\hline Total & & 92 & & 91 & & 86 & \\
\hline
\end{tabular}

enfermedades con potencial zoonótico: la influenza porcina, la hepatitis E y la infección por hantavirus, las cuales obtuvieron el mayor puntaje en la priorización por lo que se proponen como prioridades para las actividades de gestión de riesgos (87).

El enfoque semicuantitativo basado en la evidencia utilizado en este estudio partió de una revisión de la literatura científica que permitió la ponderación independiente de los criterios para cada categoría, con el fin de establecer el valor del riesgo para cada enfermedad viral; el método puede aplicarse a otros ejercicios de clasificación de las zoonosis parasitarias o bacterianas (88).

Debido a la escasez de los datos disponibles, es necesario aplicar un enfoque que recurra a la opinión de expertos para obtener resultados consensuados que obtengan el respaldo de las partes interesadas en la priorización (89-91). Por ello, el presente estudio se debe continuar con la confrontación de los resultados obtenidos y con la búsqueda del consenso de los expertos.
Los animales domésticos que comparten enfermedades con los animales silvestres pueden tener un impacto negativo en la salud humana, en la economía y en la conservación de la fauna silvestre, así como en el mantenimiento de una infección. Por otra parte, las enfermedades compartidas representan una carga significativa para la salud pública, las economías mundiales y la conservación de la biodiversidad (92), en tanto que las enfermedades virales representan una amenaza para la eficiencia de los sistemas productivos, ya que los virus que infectan a los cerdos domésticos también son capaces de infectar a los cerdos silvestres (93).

Es importante tener en cuenta los aspectos que se refieren a la naturaleza de la infección individual en los animales, la dinámica de la infección en la población y su distribución geográfica, así como la interacción de los reservorios de vida silvestre con los animales domésticos. La presentación de la enfermedad en cada especie involucrada debe ser examinada, así como las interacciones entre el medio natural y las especies domésticas (94). 
Cuadro 4. Puntuación baja de enfermedades virales

\begin{tabular}{|c|c|c|c|c|c|c|c|}
\hline Categoría & Criterio & $\begin{array}{c}\text { Peste porcina } \\
\text { africana }\end{array}$ & Referencia & PRRS & Referencia & $\begin{array}{l}\text { Enfermedad } \\
\text { de Aujeszky }\end{array}$ & Referencia \\
\hline \multirow[t]{8}{*}{ Epidemiología } & 1. Tasa de morbilidad & 7 & (61) & 7 & $(70)$ & 3 & $(74)$ \\
\hline & 2. Tasa de mortalidad & 7 & (61) & 7 & (70) & 3 & (74) \\
\hline & 3. Especificidad del patógeno & 5 & (62) & 7 & $(70)$ & 4 & $(74)$ \\
\hline & 4. Modo de transmisión & 7 & (63) & 7 & $(56)$ & 7 & (75) \\
\hline & 5. Persistencia en el medio ambiente & 3 & (56) & 6 & (71) & 6 & (76) \\
\hline & 6. Enfermedad clínica en cerdos & 4 & (63) & 7 & (56) & 2 & (77) \\
\hline & 7. Enfermedad clínica en animales silvestres & 7 & (64) & 6 & (56) & 6 & (78) \\
\hline & 8. Evidencia circulación en Colombia & 1 & (62) & 4 & (72) & 1 & (79) \\
\hline \multirow{3}{*}{$\begin{array}{l}\text { Prevención/ } \\
\text { control }\end{array}$} & 1. Control de reservorio/ vector & 6 & (65) & 1 & (70) & 1 & (80) \\
\hline & 2. Vacunación & 1 & (66) & 0 & & 1 & (75) \\
\hline & 3. Tratamiento & 6 & (66) & 3 & (73) & 7 & (81) \\
\hline \multirow{4}{*}{$\begin{array}{l}\text { Economía y } \\
\text { comercio }\end{array}$} & 1. Disminución de la productividad & 5 & (67) & 4 & (73) & 6 & (82) \\
\hline & 2. Limitaciones de importación-exportación & 1 & $(56)$ & 5 & (73) & 7 & (83) \\
\hline & 3. Impacto en sectores adyacentes (turismo) & 4 & (67) & 3 & $(56)$ & 4 & (82) \\
\hline & 6. Impacto zoonótico (costo de la enfermedad) & 1 & (68) & 0 & & 0 & \\
\hline \multirow[t]{9}{*}{ Salud pública } & 1. Zoonosis & 0 & & 0 & & 0 & (84) \\
\hline & 2. Clasificación de la zoonosis & 0 & & 0 & & 0 & (84) \\
\hline & 3. Enfermedad conocida en humanos & 0 & & 0 & & 0 & (84) \\
\hline & 4. Tasa de morbilidad & 0 & & 0 & & 0 & (79) \\
\hline & 5. Tasa de mortalidad & 0 & & 0 & & 0 & (79) \\
\hline & 6. Modo de transmisión & 0 & & 0 & & 0 & (79) \\
\hline & 7. Presencia de plan de control & 0 & & 0 & & 0 & (79) \\
\hline & 8. Vacunación & 0 & & 0 & & 0 & (79) \\
\hline & 9. Tratamiento & 0 & & 0 & & 0 & (79) \\
\hline \multirow[t]{3}{*}{ Sociedad } & 1. Disminución del consumo & 3 & (69) & 2 & $(56)$ & 4 & (80) \\
\hline & 2. Percepción del problema por parte del consumidor & 4 & (67) & 2 & (73) & 2 & (82) \\
\hline & 3. Impacto en animales silvestres y la biodiversidad & 6 & (67) & 5 & (56) & 5 & (82) \\
\hline Total & & 77 & & 76 & & 69 & \\
\hline
\end{tabular}

PRRS: Porcine Reproductive and Respiratory Syndrome

Cuadro 5. Valores de los criterios de evaluación de las enfermedades virales

\begin{tabular}{lc}
\hline Enfermedad & $\begin{array}{c}\text { Suma de } \\
\text { criterios }\end{array}$ \\
\hline Valor alto & \\
1. Influenza porcina & 133 \\
2. Hepatitis E & 123 \\
3. Infección por hantavirus & 103 \\
Valor intermedio & \\
4. Peste porcina clásica & 92 \\
5. Circovirosis & 91 \\
6. Parvovirosis & 86 \\
Valor bajo & \\
7. Peste porcina africana & 77 \\
8. Síndrome reproductivo y respiratorio porcino & 76 \\
9. Enfermedad de Aujeszky & 69 \\
\hline
\end{tabular}

En este sentido, Corner (94) indica que una población de animales infectados se puede clasificar como huésped de mantenimiento o diseminadora del virus, dependiendo de la dinámica de la infección. En los reservorios la infección puede persistir por transmisión intraespecífica y, también, puede ser fuente de infección para otras especies. El estado de mantenimiento y propagación del huésped determina si es necesario el control de cada especie de huéspedes para la prevención y el control de la enfermedad, y para la predicción de la presencia continua de la infección en la especie una vez que se elimine la fuente de infección. Sin embargo, la demografía y el comportamiento de las poblaciones desempeñan un papel importante en la transmisión de patógenos intraespecíficos e interespecificos, y determinan las tasas de contacto y la exposición al ambiente (95).

A este respecto, la densidad de la población de cerdos silvestres en el ámbito mundial está en ascenso, lo que significa un mayor número de reservorios disponibles para diseminar la enfermedad, así como altas tasas de contacto entre huéspedes (96). En cuanto al papel del cerdo silvestre como reservorio de enfermedades virales y de los cerdos domésticos en las enfermedades priorizadas (influenza porcina, hepatitis $\mathrm{E}$ y hantavirus), las características ecológicas y la distribución de agentes virales son de importancia para las intervenciones en salud pública; además, los métodos para priorizar las situaciones de salud humana y animal son un instrumento útil para comprender mejor los efectos de las enfermedades virales en la dinámica de la población de cerdos 
Cuadro 6. Principales enfermedades priorizadas según la categoría con mayor puntación de criterios

\begin{tabular}{|c|c|c|c|c|}
\hline Categoría & Criterio & Influenza porcina & Hepatitis E & Infección por hantavirus \\
\hline \multirow[t]{8}{*}{ Epidemiología } & 1. Tasa de morbilidad & 7 & 7 & 0 \\
\hline & 2. Tasa de mortalidad & 6 & 1 & 0 \\
\hline & 3. Especificidad del patógeno & 7 & 7 & 5 \\
\hline & 4. Modo de transmisión & 7 & 7 & 7 \\
\hline & 5. Persistencia en el medio ambiente & 7 & 2 & 7 \\
\hline & 6. Enfermedad clínica en cerdos & 7 & 7 & 2 \\
\hline & 7. Enfermedad clínica en animales silvestres & 7 & 4 & 5 \\
\hline & 8. Evidencia circulación en Colombia & 7 & 6 & 5 \\
\hline \multirow[t]{9}{*}{ Salud pública } & 1. Zoonosis & 7 & 7 & 7 \\
\hline & 2. Clasificación de la zoonosis & 0 & 7 & 0 \\
\hline & 3. Enfermedad conocida en humanos & 7 & 7 & 6 \\
\hline & 4. Tasa de morbilidad & 2 & 5 & 6 \\
\hline & 5. Tasa de mortalidad & 6 & 2 & 5 \\
\hline & 6. Modo de transmisión & 7 & 5 & 7 \\
\hline & 7. Existencia de plan de control & 2 & 4 & 5 \\
\hline & 8. Vacunación & 1 & 1 & 1 \\
\hline & 9. Tratamiento & 2 & 7 & 2 \\
\hline
\end{tabular}

silvestres y la circulación de agentes patógenos en las poblaciones de cerdos domésticos y de humanos (93).

Si bien es cierto que la influenza porcina se considera una zoonosis, y que los cerdos pueden actuar como un reservorio intermedio para el virus de la influenza aviar, potencialmente infeccioso para los humanos, es necesario comprender la interacción de los cerdos silvestres, los cerdos domésticos y los seres humanos en el caso de este tipo de virus, el cual, además, se relaciona con las particularidades del sistema inmunitario porcino (97).

En el contexto nacional, el Instituto Nacional de Salud, entidad encargada de operar y fortalecer el sistema de vigilancia y análisis del riesgo en salud pública, vigila enfermedades de impacto en la salud pública que el hombre comparte con los animales como son los accidentes ofídicos y causados por animales ponzoñosos, la rabia y las agresiones de animales potencialmente transmisores de rabia, la brucelosis, las enfermedades priónicas, las encefalitis equinas, la leptospirosis, la peste, la rabia y el tifus, pero las tres enfermedades zoonóticas priorizadas no están sujetas a esta vigilancia (98).

En cuanto al virus animal de la hepatitis $E$, identificado en los cerdos domésticos, en los cerdos silvestres y en los ciervos, su potencial zoonótico ha generado una gran preocupación en los países industrializados y en desarrollo. Es claro que la mayoría de las infecciones por el virus de la hepatitis E, no solo se adquieren por viajar a zonas donde es endémica. La infección por este virus se consideró inicialmente endémica, principalmente en los países en desarrollo; sin embargo, hoy se sabe que es la presentación de hepatitis viral más grave en todo el mundo por su naturaleza zoonótica, a lo que se añaden los casos recientemente reportados de infección crónica, por lo cual se requiere el control de este virus (99).

Los hantavirus, reportados recientemente en el continente americano, son otro ejemplo de agentes zoonóticos que causan morbilidad y mortalidad en humanos y requieren respuestas rápidas y oportunas basadas en el trabajo interdisciplinario de médicos, veterinarios y biólogos (100).

El conocimiento de las enfermedades que circulan en poblaciones de vida silvestre puede ser importante, no solo para la conservación de la fauna y la producción de cerdos domésticos, sino también para la salud pública, ya que las enfermedades zoonóticas plantean una importante amenaza para la salud humana y, por lo tanto, deben tenerse en cuenta por ser causantes de enfermedades graves y de la disminución o pérdida de la producción en los animales productores de alimentos, o de mortalidad en los animales silvestres, lo que puede conducir a la disminución de especies y al desequilibrio ecológico (101).

En Colombia, el sistema de vigilancia de las zoonosis adolece de un subregistro de casos humanos de zoonosis, así como de la falta de políticas de salud pública veterinaria, y de sistemas de vigilancia epidemiológica débiles y fragmentados. La falta de redes de laboratorios (102) es un agravante para la identificación y el tratamiento de 
las enfermedades virales en la interfaz de cerdos silvestres, cerdos domésticos y seres humanos. La transmisión de microorganismos patógenos puede darse como resultado del movimiento de animales silvestres y animales domésticos entre hábitats, lo cual ocurre en sitios en los que convergen estas especies con el hombre, en áreas protegidas o en áreas comunes que constituyen un punto de confluencia de patógenos oportunistas involucrados en la presentación de enfermedades, y cuya composición, diversidad y densidad afectan el desarrollo natural de las especies en cuanto a la interacción con su entorno. De ahí la importancia de entender esta interfaz y el mecanismo de transmisión de patógenos en las áreas comunes para los animales domésticos, los animales silvestres y los seres humanos, con el fin de analizar el riesgo local y global que implican (103).

La relación entre la salud y la enfermedad en los humanos, los animales y los ecosistemas, y sus implicaciones en la transmisión de las enfermedades zoonóticas, requieren de un enfoque coordinado y colaborativo, multidisciplinario e intersectorial en el que los sectores involucrados compartan responsabilidades, y aporten su conocimiento para identificar y disminuir las enfermedades humanas y animales, apoyados en el intercambio de información sobre estas, su epidemiología y los riesgos que entrañan (104).

En el presente estudio se proporciona un instrumento útil para identificar y priorizar las enfermedades virales en la interfaz de cerdos silvestres, cerdos domésticos y seres humanos, así como de zoonosis importantes, y para priorizarlas. Los métodos semicuantitativos son útiles frente a las múltiples dimensiones y ajustes de prioridades que deben cuantificarse y valorarse. El estudio, no obstante, tiene limitaciones debido a los pocos datos disponibles y a la naturaleza inespecífica del diagnóstico clínico. De todas maneras, la evaluación de los datos reportados suministra información de calidad para una mejor comprensión de los riesgos en salud pública, lo cual es de utilidad para asignar recursos en países en desarrollo. Si bien es cierto que los recursos económicos son limitados, podrían generar beneficios en investigaciones e intervenciones de enfermedades que a menudo se encuentran desatendidas. El método propuesto tiene limitaciones por el gran número de enfermedades comunes en los cerdos silvestres que no se analizan, pues no se hace vigilancia de estos animales y la detección de signos clínicos o la confirmación de una enfermedad es un proceso dispendioso $(105,106)$.

Si bien el Instituto Colombiano Agropecuario diseña y ejecuta programas oficiales para controlar y erradicar enfermedades endémicas de prioridad nacional que involucran a estas especies, la principal enfermedad porcina vigilada es la peste porcina clásica (107).

Los métodos semicuantitativos para priorizar enfermedades aportan datos imparciales para la toma de decisiones, pero se utilizan poco en los países en desarrollo por la carencia de datos de la vigilancia en salud pública. El control de enfermedades compartidas con la vida silvestre requiere el desarrollo de estrategias que reduzcan la transmisión de agentes patógenos entre la vida silvestre, los animales domésticos y los seres humanos. La vigilancia sanitaria de la fauna silvestre es tan relevante como la de los animales domésticos, y debe llevarse a cabo mediante la colaboración de los sectores encargados del control y la prevención de enfermedades zoonóticas.

En el contexto actual de variabilidad y cambio climático, desforestación, aumento de las especies amenazadas, sobrepoblación, expansión de las fronteras agrícolas, pecuarias y urbanísticas, y problemas que afectan a todo el mundo, es necesario adoptar una visión diferente, enfocada en las nuevas tendencias impuestas en las prácticas de producción animal por la globalización de la industria alimentaria. Teniendo en cuenta las situaciones sociales en las que son cada vez más acentuados los cambios demográficos, como aumento de la población, desplazamiento, movilización y urbanización, así como los cambios en los patrones de las poblaciones de animales silvestres, se deben implementar mejores sistemas de vigilancia epidemiológica, y nuevos enfoques para el control y la prevención en el marco de la relación entre la salud animal y la humana.

\section{Conflicto de intereses}

Los autores declaramos que no tenemos intereses económicos o personales relacionados con los resultados publicados en este artículo.

\section{Financiación}

Este estudio fue financiado con recursos del semillero de investigación "Una salud" y el Grupo de Epidemiología y Salud Pública de la Universidad de La Salle. 


\section{Referencias}

1. Barasona JA, Latham MC, Acevedo P, Armenteros JA, Latham AD, Gortázar C, et al. Spatiotemporal interactions between wild boar and cattle: Implications for cross-species disease transmission. Vet Res. 2014;45:1-122. http://dx.doi. org/10.1186/s13567-014-0122-7

2. Gortázar C, Díez-Delgado I, Barasona JA, Vicente J, De La Fuente J, Boadella M. The wild side of disease control at the wildlife-livestock-human interface: A review. Front Vet Sci. 2015;1:27. http://dx.doi.org/10.3389/fvets.2014.00027

3. Gortázar C, Ferroglio E, Höfle U, Frölich K, Vicente J. Diseases shared between wildlife and livestock: A European perspective. Eur J Wild Res. 2007;53:241-56. http://dx.doi. org/10.1007/s10344-007-0098-y

4. Monsalve S, Mattar S, González M. Zoonosis transmitidas por animales silvestres y su impacto en las enfermedades emergentes y reemergentes. Rev MVZ Córdoba. 2009;14: 1762-73.

5. Logan-Rist C, Arriola C, Rubin C. Prioritizing zoonoses: A proposed one health tool for collaborative decision-making. Centers for Disease Control and Prevention. PLOS ONE. 2014;9:e109986. http://dx.doi.org/10.1371/journal.pone. 0109986

6. Jones K, Patel N, Levy M, Storeygard A. Global trends in emerging infectious disease. Nature. 2008;451:990-3. http:// dx.doi.org/10.1038/nature06536.

7. Jones B, McKeever D, Grace D, Pfeiffer D, Mutua F, Njuki $J$, et al. Zoonoses (Project 1). Wildlife/domestic livestock interactions. Nairobi: International Livestock Research Institute; 2011. p. 128

8. Humblet MF, Vandeputte S, Albert A, Gosset C, Kirschvink $\mathrm{N}$, Haubruge $\mathrm{E}$, et al. Multidisciplinary and evidence-based method for prioritizing diseases of food-producing animals and zoonoses. Emerg Infect Dis. 2012;18. http://dx.doi. org/10.3201/eid1804.111151

9. Cito F, Rijks J, Rantsios A, Cunningham A, Baneth G, Guardabassik L, et al. Prioritization of companion animal transmissible diseases for policy intervention in Europe. J Comp Pathol. 2015. http://dx.doi.org/10.1016/j. jсра.2015.01.007

10. Arbeláez G, Calderón D, Rincón M, Lora A, Mercado M. Improvement of two diagnostics methods for detection of influenza swine virus. Univ Sci. 2008;3:65-74.

11. Vittecoq M, Grandhomme V, Simon G, Herve S, Blanchon T, Renaud F, et al. Study of influenza A virus in wild boars living in a major duck wintering site. Infect Genet Evol. 2012;12:483-6. http://dx.doi.org/10.1016/j.meegid.2011. 12.003

12. Brown I. The epidemiology and evolution of influenza viruses in pigs. Vet Microbiol. 2000;74 29-46. http://dx.doi. org/10. 1016/S0378-1135(00)00164-4

13. Baratelli M, Córdoba L, Pérez L, Maldonado J, Frailea L, Núñez J, et al. Genetic characterization of influenza a viruses circulating in pigs and isolated in north-east Spain during the period 2006-2007. Res Vet Sci. 2014;96:380-8. http://dx.doi.org/10.1016/j.rvsc.2013.12.006

14. Hanssen $\mathbf{H}$, Hincapié $\mathbf{O}$, López J. Influenza en porcinos de Antioquia, Colombia. Bol Oficina Sanit Panam. 1977;82: 35-43.
15. Rajao D, Anderson T, Gauger P, Vincent A. Pathogenesis and vaccination of influenza $A$ virus in swine. Curr Top Microbiol Immunol. 2014;1:307-26. http://dx.doi.org/10. 1007/82_2014_391

16. Lester P, Perera C, Coronado L, Ríos L, Vega A, Frías M, et al. Molecular epidemiology study of swine influenza virus revealing a reassorted virus $\mathrm{H} 1 \mathrm{~N} 1$ in swine farms in Cuba. Prev Vet Med. 2015;119:172-8. http://dx.doi.org/10.1016/j. prevetmed.2015.02.013

17. Alfonso P. Amenazas y oportunidades de reducción de riesgos por virus influenza en la interface animal-hombre. Rev Salud Anim. 2010;32:1-10.

18. Calore E, Uipc D, Pérez N. Pathology of the swine-origin influenza A (H1N1) flu. Pathol Res Pract. 2011;207:86-90. http://dx.doi.org/10.1016/j.prp.2010.11.003

19. Kumar S, Subhadra S, Singh B, Panda B. Hepatitis E virus: The current scenario. Int J Infect Dis. 2012;17:228-33. http://dx.doi.org/10.1016/j.jij.2012.11.026

20. Di Bartolo I, Angeloni G, Ponterio E, Ostanello F, Ruggeri F. Detection of hepatitis $E$ virus in pork liver sausages. Int J Food Microbiol. 2015;193:29-33. htt http://dx.doi. org/10.1016/j.ijfoodmicro.2014.10.005

21. Martelli F, Caprioli A, Zengarini M, Marata A, Fiegna C, Di Bartolo I, et al. Detection of hepatitis E virus (HEV) in a demographic managed wild boar (Sus scrofa scrofa) population in Italy. Vet Microbiol. 2008;26:74-81. http:// dx.doi.org/10.1016/j.vetmic.2007.07.004

22. Nilsa D, Seminati C, Pina S, Mateu E, Martín M, Segalés $J$. Detection of hepatitis $E$ virus in liver, mesenteric lymph node, serum, bile and faeces of naturally infected pigs affected by different pathological conditions. Vet Microbiol. 2007;119:105-14. http://dx.doi.org/10.1016/j.vetmic.2006. 08.027

23. Banks M, Bendall R, Grierson S, Heath G, Mitchell J, Dalton H. Human and porcine hepatitis $E$ virus strains, United Kingdom. Emerg Infect Dis. 2004;10:953-5. http:// dx.doi.org/10.3201/eid1005.030908

24. Gutiérrez-Vergara C, Quintero J, Duarte JF, Suescún JP, López-Herrera A. Detection of hepatitis $\mathrm{E}$ virus genome in pig livers in Antioquia, Colombia. Genet Mol Res. 2015;14:2890-1. http://dx.doi.org/10.4238/2015.March.31.20

25. Sanford B J, Opriessnig T, Kenney SP, Dryman BA, Córdoba L, Meng XJ. Assessment of the cross-protective capability of recombinant capsid proteins derived from pig, rat, and avian hepatitis E viruses (HEV) against challenge with a genotype $3 \mathrm{HEV}$ in pigs. Vaccine. 2012;30:6249-55. http://dx.doi.org/10.1016/j.vaccine.2012.08.013

26. Song W, Xianfeng C, Xing D, Chen D, Mingjie X, Jiuhong $\mathrm{L}$, et al. Rabbit and human hepatitis $\mathrm{E}$ virus strains belong to a single serotype. Virus Res. 2013;6:101-6. http://dx.doi. org/10.1016/j.virusres.2013.05.013

27. Montalvo M, Canwat-Owot J, Corrreia B, Bello M, Flaquet $\mathrm{P}$, Sariego S, et al. Hepatitis E virus genotype 3 in humans and swine, Cuba. Infect Genet Evol. 2013;14:335-9. http:// dx.doi.org/10.1016/j.meegid.2012.12.022

28. Oliveira-Filho EF, Bank-Wolf BR, Thiel HJ, König M. Phylogenetic analysis of hepatitis $\mathrm{E}$ virus in domestic swine and wild boar in Germany. Vet Microbiol. 2014;174:233-8. http://dx.doi.org/10.1016/j.vetmic.2014.09.011 
29. Quintana-González A. Virus de la hepatitis E. Rev Biomed. 2003;14:165-89.

30. Ramos C. Los hantavirus causantes de la fiebre hemorrágica con síndrome renal y del síndrome pulmonar. Salud Pública Méx. 2008;50:334-40.

31. Harkness JE, Turner PV, Vandewoude S, Wheler CL. Harkness and Wagner's Biology and Medicine of Rabbits and Rodents. Fifth edition. Hoboken, NJ: John Wiley \& Sons; 2013.

32. Hart C, Bennett M. Hantavirus infections: Epidemiology and pathogenesis. Microbes Infect. 1999;1:1229-37. http:// dx.doi.org/10.1016/S1286-4579(99)00238-5

33. Mitchell M, Tully TN Jr. Zoonotic diseases. En: Quesenberry K, Carpenter J, editors. Ferrets, Rabbits and Rodents. Third edition. St. Louis, MO: Elsevier; 2012. p. 557-65. http:// dx.doi.org/10.1016/B978-1-4160-6621-7.18001-7

34. Cook R, Karesh W. Emerging diseases at the interface of people, domestic animals, and wildlife. En: Miller E, Fowler $M$, editors. Fowler's zoo and wild animal medicine current therapy. First edition. St. Louis, MO: Elsevier Saunders; 2011. p. 136-46. http://dx.doi.org/10.1016/B978-1-43771986-4. 00018-4

35. Boklund A, Goldbach S, Uttenthal A, Alban L. Simulating the spread of classical swine fever virus between a hypothetical wild-boar population and domestic pig herds in Denmark. Prev Vet Med. 2008;85:187-206. http://dx.doi. org/10.1016/j.prevetmed.2008.01.012

36. Moennig V, Floegel-Niesmann G, Greiser-Wilke I. Clinical signs and epidemiology of classical swine fever: A review of new knowledge. Vet J. 2003;165:11-20. http://dx.doi. org/10.1016/S1090-0233(02)00112-0

37. Ferrer E, Fonseca O, Percedo M, Abeledo M. Classical swine fever in the Americas and the Caribbean. Present situation and prospects for control and eradication. Rev Salud Anim. 2010;32:11-21.

38. Sawford K, Geong M, Bulu P, Drayton E, Mahardika G, Leslie E, et al. An investigation of classical swine fever virus seroprevalence and risk factors in pigs in East Nusa Tenggara, eastern Indonesia. Prev Vet Med. 2015;119:190202. http://dx.doi.org/10.1016/j.prevetmed.2015.02.002

39. Instituto Colombiano Agropecuario. Colombia, Sanidad Animal, 2005. Fecha de consulta: 24 de mayo de 2015. Disponible en: http://www.ica.gov.co/getattachment/4b322f441380-4481-84c9-cf5078cd14f8/1.aspx

40. Simon G, Le Dimna M, Le Potier MF, Pol F. Molecular tracing of classical swine fever viruses isolated from wild boars and pigs in France from 2002 to 2011. Vet Microbiol. 2013:166:631-8. http://dx.doi.org/10.1016/j.vetmic.2013. 06.032

41. Blome S, Grotha I, Moennig V, Greiser-Wilke I. Classical swine fever virus in South-Eastern Europe -Retrospective analysis of the disease situation and molecular epidemiology. Vet Microbiol. 2010;146:276-84. http://dx.doi.org/10.1016/j. vetmic.2010.05.035

42. Lévai R, Barna T, Fábián K, Blome S, Belák K, Bálint Á, et al. Pre-registration efficacy study of a novel marker vaccine against classical swine fever on Maternally Derived Antibody negative (MDA-) target animals. Biologicals. 2015;43:92-9. http://dx.doi.org/10.1016/j.biologicals.2014.12.004
43. Alarcón P, Rushton J, Wieland B. Cost of post-weaning multi-systemic wasting syndrome and porcine circovirus type-2 subclinical infection in England -An economic disease model. Prev Vet Med. 2013;110:88-102. http://dx. doi.org/10.1016/j.prevetmed.2013.02.010

44. Darwich L, Mateu E. Immunology of porcine circovirus type 2 (PCV2). Virus Res. 2012;164:61-7. http://dx.doi. org/10.1016/j.virusres.2011.12.003

45. Pujols J, Lorca-Oró C, Díaz C, Russell L, Campbell J, Crenshaw D, et al. Commercial spray-dried porcine plasma does not transmit porcine circovirus type 2 in weaned pigs challenged with porcine reproductive and respiratory syndrome virus. Vet J. 2011:190:e16-20. http://dx.doi. org/10.1016/j.tvjl.2011.02.021

46. Knell S, Willems H, Hertrampf B, Reine G. Comparative genetic characterization of porcine circovirus type 2 samples from German wild boar populations. Vet Microbiol. 2005;109:169-77. http://dx.doi.org/10.1016/j.vetmic.2005. 06.004

47. Díaz I, Cortey M, Darwich L, Sibila M, Mateu E, Segalés J. Subclinical porcine circovirus type 2 infection does not modulate the immune response to an Aujeszky's disease virus vaccine. Vet J. 2012;194:84-8. http://dx.doi. org/10.1016/j.tvjl.2012.02.014

48. Grau-Roma L, Fraile L, Segalés J. Recent advances in the epidemiology, diagnosis and control of diseases caused by porcine circovirus type 2 . Vet J. 2011;187:23-32. http:// dx.doi.org/10.1016/j.tvjl.2010.01.018

49. Vidigal P, Mafra CL, Silva FM, Fietto JL, Silva-Júnior A, Almeida MR. Tripping over emerging pathogens around the world: A phylogeographical approach for determining the epidemiology of porcine circovirus-2 (PCV-2), considering global trading. Virus Res. 2012;163:320-7. http://dx.doi. org/10.1016/j.virusres.2011.10.019

50. Rose N, Eveno N, Grasland B, Nignol A, Oger A, Jestin A, et al. Individual risk factors for Post-weaning Multisystemic Wasting Syndrome (PMWS) in pigs: A hierarchical Bayesian survival analysis. Prev Vet Med. 2009;90:168-79. http://dx. doi.org/10.1016/j.prevetmed.2009.04.010

51. Burbelo P, Ragheb JA, Kapoor A, Zhang Y. The serological evidence in humans supports a negligible risk of zoonotic infection from porcine circovirus type 2 . Biologicals. 2013;41:430-4. http://dx.doi.org/10.1016/j. biologicals.2013.09.005

52. Hattermann K, Roedner C, Schmitt C, Finsterbusch T, Steinfeldt T, Mankertz A. Infection studies on human cell lines with porcine circovirus type 1 and porcine circovirus type 2. Xenotransplantation. 2004;11:284-94. http://dx.doi. org/10.1111/j.1399-3089.2004.00134.x

53. Roca M, Gimeno M, Bruguera S, Segalés J, Díaz I, Galindo-Cardiel IJ, et al. Effects of challenge with a virulent genotype II strain of porcine reproductive and respiratory syndrome virus on piglets vaccinated with an attenuated genotype I strain vaccine. Vet J. 2012;193:92-6. http://dx. doi.org/10.1016/j.tvjl.2011.11.019

54. Mengeling W, Lager K, Vorwald A. The effect of porcine parvovirus and porcine reproductive and respiratory syndrome virus on porcine reproductive performance. Anim Reprod Sci. 2000;60:199-210. http://dx.doi.org/10.1016/ S0378-4320(00)00135-4 
55. Borrallo G, Guillermo M, Sánchez J. Parvovirosis porcina. Mundo Ganadero. 1993;11:65-8.

56. Ruíz-Fonz F, Segalés J, Gortázar $\mathbf{C H}$. A review of viral diseases of the European wild boar: Effects of population dynamics and reservoir role. Vet J. 2007;176:158-69. http:// dx.doi.org/10.1016/j.tvjl.2007.02.017

57. Cadar D, Kiss T, Ádám D, Cságola A, Novosel D, Tuboly T. Phylogeny, spatio-temporal phylodynamics and evolutionary scenario of Torque teno sus virus 1 (TTSuV1) and 2 (TTSuV2) in wild boars: Fast dispersal and high genetic diversity. Vet Microbiol. 2013;166:200-13. http:// dx.doi.org/10.1016/j.vetmic.2013.06.010

58. Fenati M, Armaroli E, Corrain R, Guberti V. Indirect estimation of porcine parvovirus maternal immunity decay in free-living wild boar (Sus scrofa) piglets by capturerecapture data. Vet J. 2009;180:262-4. http://dx.doi. org/10.1016/j.tvjl.2007.12.009

59. Rico S, Molina S, Pabón F. Detección y aislamiento del parvovirus porcino en Medellín, Colombia. Rev Col Cienc Pec. 2003;16:40-4.

60. Antonis A, Bruschke C, Rueda P, Maranga L, Casal $\mathbf{J}$, Vela $\mathbf{C}$, et al. A novel recombinant virus-like particle vaccine for prevention of porcine parvovirus-induced reproductive failure. Vaccine. 2006;24:5481-90. http://dx. doi.org/10.1016/j.vaccine.2006.03.089

61. Carvalho H, Zúquete $S$, Wijnveld $M$, Weesendorp $\mathbf{E}$, Jongejan $\mathbf{F}$, Stegeman $\mathbf{A}$, et al. No evidence of African swine fever virus replication in hard ticks. Ticks Tick Borne Dis. 2014;5:582-9. http://dx.doi.org/10.1016/j. ttbdis.2013.12.012

62. Blome S, Gabriel C, Beer M. Pathogenesis of African swine fever in domestic pigs and European wild boar. Virus Res. 2013;173:122-30. http://dx.doi.org/10.1016/j. virusres.2012.10.026

63. Blome S, Gabriel C, Beer M. Modern adjuvants do not enhance the efficacy of an inactivated African swine fever virus vaccine preparation. Vaccine. 2014;32:3879-82. http:// dx.doi.org/10.1016/j.vaccine.2014.05.051

64. Sánchez-Vizcaíno JM, Mur L, Martínez-López B. African swine fever: An epidemiological update. Transbound Emerg Dis. 2012;59:27-35. http://dx.doi.org/10.1111/j.18651682.2011.01293.x

65. Gogin A, Gerasimov V, Malogolovkin A, Kolbasov D. African swine fever in the North Caucasus region and the Russian Federation in years 2007-2012. Virus Res. 2013;173:198-203. http://dx.doi.org/10.1016/j.virusres.2012. 12.007

66. Oura C, Edwards L, Batten C. Virological diagnosis of African swine fever-Comparative study of available tests. Virus Res. 2013;173:150-8. http://dx.doi.org/10.1016/j. virusres.2012.10.022

67. Gavier-Widén D, Gortázar C, Stahl K, Neimanis AS, Rossi S, Hard AF Segerstad C, et al. African swine fever in wild boar in Europe: A notable challenge. Vet Rec. 2015;176:199-200. http://dx.doi.org/10.1136/vr.h699

68. World Organisation for Animal Health (OIE). Peste porcina africana. Fecha de consulta: 28 de abril de 2015. Disponible en: http://www.oie.int/doc/ged/D13954.PDF
69. Ruiz-Fons F. A review of the current status of relevant zoonotic pathogens in wild swine (Sus scrofa) populations: Changes modulating the risk of transmission to humans. Transbound Emerg Dis. 2015. http://dx.doi.org/10.1111/ tbed.12369

70. Schelkopf A, Nerem J, Cowles B, Amodie D, Swalla R, Dee R. Reproductive, productivity, and mortality outcomes in late-gestation gilts and their litters following simulation of inadvertent exposure to a modified-live vaccine strain of porcine reproductive and respiratory syndrome (PRRS) virus. Vaccine. 2014;32:4639-43. http://dx.doi.org/10.1016/j. vaccine.2014.06.073

71. Sutherland $\mathbf{M}$, Niekamp S, Johnson R, van Alstine W, Salak-Johnson J. Heat and social rank impact behavior and physiology of PRRS-virus-infected pigs. Physiol Behav. 2006;90:73-81. http://dx.doi.org/10.1016/j. physbeh.2006.08.029

72. Cruz MC. Prevalencia serológica del síndrome reproductivo y respiratorio porcino (PRRS) en cerdos de explotaciones extensivas de Colombia. Rev Med Vet Zoot. 2006;53:33-41.

73. Food and Agriculture Organization, FAO. EMPRES Boletín de enfermedades transfronterizas de los animals. Fecha de consulta: 28 de abril de 2015. Disponible en: http://www.fao.org/docrep/011/i0574s/i0574s00.HTM

74. Verpoest S, Brigitte C, De Regge N. Molecular characterization of Belgian pseudorabies virus isolates from domestic swine and wild boar. Vet Microbiol. 2014;172:72-7. http://dx.doi.org/10.1016/j.vetmic.2014.05.001

75. Maresch C, Lange E, Teifke J, Fuchs W, Klupp B, Müller $\mathrm{T}$, et al. Oral immunization of wild boar and domestic pigs with attenuated live vaccine protects against Pseudorabies virus infection. Vet Microbiol. 2012;161:20-5. http://dx.doi. org/10.1016/j.vetmic.2012.07.002

76. Del Rincón J. Enfermedad de Aujeszky: descripción y consideraciones. Mundo Ganadero. 1996;81:42-4.

77. Gerdts V, Jöns A, Mettenleiter T. Potency of an experimental DNA vaccine against Aujeszky's disease in pigs. Vet Microbiol. 1999;66:1-13. http://dx.doi.org/10.1016/ S0378-1135(98)00300-9

78. Albina E, Mesplède A, Chenut G, Le Potier M, Bourbao G, Le Gal S, et al. A serological survey on classical swine fever (CSF), Aujeszky's disease (AD) and porcine reproductive and respiratory syndrome (PRRS) virus infections in French wild boars from 1991 to 1998. Vet Microbiol. 2000;77:43-57. http://dx.doi.org/10.1016/S0378-1135(00)00255-8

79. Rodríguez D, Naranjo J, Rincón M, Peña M. Estudio serológico de Aujeszky en las principales regiones porcícolas del país. Porcicultura Colombiana. 2013;2:24-7.

80. Gillespie RR, Hill MA. Infection of pigs by aerosols of Aujeszky's disease virus and their shedding of the virus. Res Vet Sci. 1996;60:228-33. http://dx.doi.org/10.1016/ S0034-5288(96)90044-2

81. Müller T, Teuffert J, Zellmer R, Conraths FJ. Experimental infection of European wild boars and domestic pigs with pseudorabies viruses with differing virulence. Am J Vet Res. 2001;62:252-8. http://dx.doi.org/10.2460/ajvr.2001.62.252

82. Moreno A, Sozzi E, Grilli G, Gibelli L, Gelmetti D, de Lelli D, et al. Detection and molecular analysis of Pseudorabies virus strains isolated from dogs and a wild boar in Italy. Vet Microbiol. 2015;177:359-65. http://dx.doi.org/10.1016/j. vetmic.2015.04.001 
83. Boklund A, Dahl J, Alban L. Assessment of confidence in freedom from Aujeszky's disease and classical swine fever in Danish pigs based on serological sampling - Effect of reducing the number of samples. Prev Vet Med. 2012;110: 214-22. http://dx.doi.org/10.1016/j.prevetmed.2012.11.027

84. Boelaert F, Deluyker H, Maes D, Godfroid J, Raskin A, Varewijck $\mathrm{H}$, et al. Prevalence of herds with young sows seropositive to pseudorabies (Aujeszky's disease) in northern Belgium. Prev Vet Med. 1999;41:239-55. http:// dx.doi.org/10.1016/S0167-5877(99)00058-6

85. Meng X, Lindsay D, Sriranganathan N. Wild boars as sources for infectious diseases in livestock and humans. Philos Trans R Soc Lond B Biol Sci. 2009;27:364;2697707. http://dx.doi.org/10.1098/rstb.2009.0086

86. Kurowicka D, Bucura C, Cooke R, Havelaar A. Probabilistic inversion in priority setting of emerging zoonoses. Risk Anal. 2010;30:715-23. http://dx.doi.org/10.1111/j.1539-6924. 2010.01378

87. Havelaar AH, van Rosse F, Bucura C, Toetenel MA, Haagsma JA. Prioritizing emerging zoonoses in the Netherlands. PLoS ONE. 2010;5:e13965. http://dx.doi. org/10.1371/journal.pone.0013965

88. Cardoen S, van Huffel X, Berkvens D, Quoilin S, Ducoffre G, Saegerman C, et al. Evidence-based semiquantitative methodology for prioritization of foodborne zoonoses. Foodborne Pathog Dis. 2009;6:1083-96. http://dx.doi. org/10.1089/fpd.2009.0291

89. Jenicek M. Epidemiology, evidenced-based medicine, and evidence-based public health. J Epidemiol. 1997;7:187-97. http://dx.doi.org/10.2188/jea.7.187

90. López J. Sobre la salud pública basada en pruebas. Rev Esp Salud Pública. 2008;82:1-4.

91. Ciliberti A, Gavier-Widén D, Yon L, Hutchings M, Artois M. Prioritisation of wildlife pathogens to be targeted in European surveillance programmes: Expert-based risk analysis focus on ruminants. Prev Vet Med. 2015;118:27184. http://dx.doi.org/10.1016/j.prevetmed.2014.11.021

92. Daszak P, Cunningham A, Hyatt $\mathbf{A}$. Emerging infectious diseases of wildlife - threats to biodiversity and human health. Science. 2000;287:443-9. http://dx.doi.org/10.1126/ science.287.5452.443

93. Ruiz-Fons F, Vicente J, Vidal D, Höfle U, Villanúa D, Gauss C, et al. Seroprevalence of six reproductive pathogens in European wild boar (Sus scrofa) from Spain: The effect on wild boar female reproductive performance. Theriogenology. 2008;65:731-43. http://dx.doi.org/10.1016/j. theriogenology.2005.07.001

94. Corner L. The role of wild animal populations in the epidemiology of tuberculosis in domestic animals: How to assess the risk. Vet Microbiol. 2006;112:303-12. http:// dx.doi.org/10.1016/j.vetmic.2005.11.015

95. Dobson A. Population dynamics of pathogens with multiple host species. Am Nat. 2004;164:64-78. http://dx.doi.org/10. $1086 / 424681$
96. Acevedo P, Vicente J, Hofle U, Cassinello J, RuizFons F, Gortázar C. Estimation of European wild boar relative abundance and aggregation: A novel method in epidemiological risk assessment. Epidemiol Infect. 2007;135:519-27. http://dx.doi.org/10.1017/S095026880 6007059

97. Mussa T, Ballester M, Silva-Campa $\mathbf{S}$, Baratelli $\mathbf{M}$, Busquets $\mathbf{N}$, Lecours $\mathbf{M}$, et al. Swine, human or avian influenza viruses differentially activates porcine dendritic cells cytokine profile. Vet Immunol Pathol. 2013;154:25-35. http://dx.doi.org/10.1016/j.vetimm.2013.04.004

98. Instituto Nacional de Salud. Enfermedades transmisibles. Fecha de consulta: 18 de junio de 2015. Disponible en: http://www.ins.gov.co/lineas-de-accion/SubdireccionVigilancia/Paginas/transmisibles.aspx\#.VmG27HYvflU

99. Abravanel F, Lhomme S, Dubois M, Peron J, Alric L, Kamar N, et al. General review hepatitis E virus. Médecine et Maladies Infectieuses. 2013;43:263-70. http://dx.doi. org/10.1016/j.medmal.2013.03.005

100. Wordl Health Organization. Future trends in veterinary public health. Geneva: WHO; 2002.

101. Cripps. P. Veterinary education, zoonoses and public health: A personal perspective. Acta Trop. 2000;76:77-80. http://dx.doi.org/10.1016/S0001-706X(00)00094-2

102. Cediel N, Villamil L, Romero J, Rentería L, Meneghi D. Setting priorities for surveillance, prevention, and control of zoonoses in Bogotá, Colombia. Rev Panam Salud Pública. 2013;33:316-24. http://dx.doi.org/10.1590/S102049892013000500002

103. Miguel E, Boulinierb T, Garine-Wichatitsky M, Carona A, Fritz H, Grosbois V. Characterizing African tick communities at a wild-domestic interface using repeated sampling protocols and models. Acta Trop. 2014;138:514. http://dx.doi.org/10.1016/j.actatropica.2014.05.019

104. Mackenzie J, Jeggo M, Daszak P, Richt J. One health: The human-animal-environment interfaces in emerging infectious diseases. The concept and examples of a one health approach. Berlin: Springer-Verlag Berlin Heidelberg; 2013. p. 365:X-362. http://dx.doi.org/10.1007/978-3-64236889-9

105. Makita K, Fèvre E, Waiswa C, Kaboyo W, Eisler M, Welburn S. Evidence-based identification of the most important livestock related zoonotic diseases in Kampala, Uganda. J Vet Med Sci. 2011;73:991-1000. http://dx.doi. org/1010.1292/jvms.11-0049

106. NG V, Sargeant JM. A quantitative approach to the prioritization of zoonotic diseases in North America: A health professionals' perspective. PloS One. 2013;8: e72172. http://dx.doi.org/10.1371/journal.pone.0072172

107. Instituto Colombiano Agropecuario. Programa de erradicación de peste porcina clásica. Fecha de consulta: 17 de junio de 2015. Disponible en: http://www.ica.gov.co/ getdoc/ea9c6aa0-a5fc-472f-869b-975b27d7ac35/PestePorcina-Clasica-(1).aspx 